\title{
Evaluation of Product Usability using Improved FP-Growth Frequent Itemset Algorithm and DSLC - FOA Algorithm for Alleviating Feature Fatigue
}

\author{
Midhunchakkaravarthy ${ }^{1}$, Divya ${ }^{2}$, Debnath Bhattacharyya ${ }^{3}$ and Tai-hoon Kim ${ }^{4 *}$ \\ ${ }^{1}$ Faculty of Computer Science and Multimedia, \\ Lincoln University College, Malaysia \\ ${ }^{2}$ Center of Postgraduate Studies, Lincoln University College, Malaysia \\ ${ }^{3}$ Dept. of Computer Science and Engineering, \\ Vignan's Institute of Information Technology, Visakhapatnam, India \\ ${ }^{4}$ Dept. of Convergence Security, Sunghin Women's University, Seoul, South Korea \\ ${ }^{1}$ midhun.research@gmail.com, 2divya.phd.research@gmail.com, \\ ${ }^{3}$ debnathb@gmail.com, ${ }^{4 * t a i h o o n n @ d a u m . n e t ~}$
}

\begin{abstract}
The development of E-commerce websites in recent years has attracted more people to buy products and services online. The development has also hooked the organizations to involve in online business processing for better growth. Generally, customers are intended to buy products with more features. Based on this traditional customer purchase behavior, the manufacturers design their product with the maximum number of features. Later, customers after using the products may get dissatisfied due to product features that are not suitable for the product. As this customer dissatisfaction is because of unwanted product features, it is termed as Feature Fatigue of a product. Nowadays customers post their opinions on E-commerce websites as reviews. For the organizations, it is the most important aspect to consider online reviews posted by existing customers. Hence those reviews may also reflect Feature Fatigue (FF) which affects the reputation and organization's growth. To solve this feature fatigue problem a novel method linear Diminishing Step and Logistic Chaos with Fruit fly Optimization Algorithm (DSLC-FOA) based Association Rule Mining (DFARM) is proposed in this paper to evaluate the product usability. In DFARM, improved Frequent Pattern - Growth (FP-Growth) Frequent Itemset algorithm has been improvised and incorporated using DSLC-FOA algorithm to evaluate the product usability. Further feature fatigue analysis is applied using Genetic Algorithm to obtain the FF Degree through usability evaluation and capability evaluation of each feature.
\end{abstract}

Keywords: Product usability, Capability evaluation, Feature Fatigue, DSLC-FOA, FPGrowth

\section{Introduction}

The customers in recent years highly depend on online purchasing where the organizations are competitive in online business platform [1]. The organizations face many challenges to compete with others in the online business. In which most important challenge is to understand customers purchase behavior [2]. This understanding can be determined through analyzing the customer reviews based on their preferences [3]. Thus, customers' reviews are an important aspect of analyzing customers purchase behavior and

Received (April 7, 2018), Review Result (May 29, 2018), Accepted (June 7, 2018)

* Corresponding Author 
provide support to make decisions in product designing. The growth of online business transactions and interest shown by the customers to purchase products online are phenomenal [4].

This involvement of online technology in the business world tends the leading manufacturers to concentrate more on online customers. The customers prefer to buy the products with more features during purchase [5]. In traditional business processes, it is imposed to add more features for increasing the Product Capability (PC), product quality and the profit [6]. But, due to the addition of many features in the products, customers are affected by high complexity in using of unwanted features of a product [7]. This results in dissatisfaction of customers on the product feature usability, which is called as Feature Fatigue (FF) [8].

The product feature usability problem will widely spread among the customers through Word Of Mouth (WOM) which affects the growth of manufacturers [9]. Hence it is highly important to remove the Feature Fatigue of a product. The major concerned areas of this research work are online product reviews, product usability, and Feature Fatigue, which are discussed briefly in the following sub-sections.

\subsection{Online Product Reviews}

These days most people are interested in using e-commerce websites to purchase the products [10]. This situation is because of proliferating e-commerce websites provided to the customers for buying the products [11]. The concept of online shopping varies from customary shopping in several behaviors. The important behavior is customers are not provided with the chances to see, realize and verify the products as they can do with customary shopping. The customers can only depend on the information provided by the manufacturers in online. To overcome this limitation the manufacturers provided the facility to the customers to share their experiences on the product usability in online with other customers [12]. The range of increasing online customers increases the habit of posting reviews about products online [4]. The reviews posted by customers in ecommerce websites about the products are like WOM [8]. Part of customers considers the reviews of existing customers as an important key in deciding to purchase the products [13]. In general, while posting the reviews customers expresses their impact on the features of a product they purchased earlier. These reviews clear out customers' opinions about the product features. Thus, it helps manufacturers to know the market opinion about their product [14].

\subsection{Product Usability}

At the time of purchase, the customers usually compare the usability of the product to make a purchase decision. Usability is the tool identify product acceptance of the customers. Also, it is the important customer requirement of a product [15] and considered as a tool to succeed in the market [16]. Usability analysis is the requirement analysis, which helps in identifying, analyzing, and satisfies customers' requirements and provides the opinions of the product to design and modify. The products with high usability provide high customer satisfaction; hence to lead in the market the manufacturers provide the products with high feature usability to satisfy the customers' requirements [16]. The product feature usability evaluation is performed to identify customer and product relationship based on usage. Thus, product feature usability evaluation is considered as an important way to improve product development based on customer requirements [8].

\subsection{Feature Fatigue}

The earlier researches show that by increasing the number of features to a product, the product capability can be increased through which product quality is also improved and final results in improved profit [12]. Even then adding more features to a product may 
create difficulty for the customer to use the product. This difficulty will result in dissatisfaction of a customer on a product, which is known as Feature Fatigue (FF) [16]. FF is an occurrence where customers tend to buy products with a maximum number of features at the time of purchasing before use, product dissatisfied after use due to unwanted features in the product. FF creates negative WOM, which can spread widely and will bring down the product and manufacturer reputation in the market. This will result in a loss of longterm customers of the organization [17]. The best method to remove FF is to minimize or remove unwanted features in a product and design the product with optimal features set of balancing Product Capability (PC) and Product Usability (PU).

\section{Literature Review}

The existing methods used to analyze online reviews, evaluate product feature usability and analyze feature fatigue are listed in the following sections.

\subsection{Online Customer Reviews and Related Theories}

The online reviews posted by the customers provide the information about products and their features availed by the organizations [18]. The count of customer reviews and customer ratings upon the product identifies the accurate aspect to be considered in increasing the purchase behavior [3]. The various existing approaches and models applied to analyze the online reviews posted by customers about the product and features upon usability are discussed in detail below.

Chong et al., [2] proposed a big data architecture for analyzing web data, where asynchronous input and output are used to request, extract and preprocess the data in realtime. The big data architecture is employed for analyzing the sentiment through applying Application Programmer Interface (API) text processing with an online classifier to process the collected reviews. Those collected reviews are then labeled with semantic values and finally, three-layered neural network modeling is applied to examine the predictors of online product sales. As a result, the online reviews are confirmed as an important predictor in online sales of a product.

Chen et al., [18] proposed three review helpfulness hypotheses, based on illustrative inferences in three aspects as reviewers, review characteristics, and several review votes. And the reasonability is verified with real-time review data. This discovers the relationship between helpfulness of a review with other aspects of online product sales. The result obtained based on the three hypotheses are, the reviews from customers, the high reviews valances and reviews with maximum votes provide helpfulness which helps in different aspects of online product sales.

Zimmermann et al., [19] proposed a framework, OPINSTREAM to identify and classify the product features based on public reviews on different products. The framework includes the processing of stream clustering, product feature extraction based on clusters, sentiment learning of each cluster. In this work, the OPINSTREAM is applied through an adoption mechanism incorporated with semi-supervised classification method for evaluation. The result determined was with better quality and time consumption upon execution.

Asghar et al., [20] proposed a rule-based approach to identify the classification of opinions from online reviews. In this approach, the product reviews are extracted based on Lexicon opinion mining. Those reviews are then classified as subjective and objective reviews. Then polarity values of subjective reviews are evaluated from the classification. Upon experimentation of this approach, the result achieved was $86 \%$ accurate on feedback $85 \%$ on reviews.

Lizhen et al., [21] proposed a vector model based on product features for effective analysis of sentiment orientation in review sentences. The model is applied with a novel feature weighting algorithm, High Adverb of Degree Count (HADC) for classifying the 
sentiment orientation based on related information in the review sentences. The HADC weighting algorithm resulted in classifying reviews at $87.5 \%$ in the least case and achieved $91 \%$ accuracy in the best case.

\subsection{Various Existing Methods of Product Usability Evaluation}

Razza et al., [22] proposed Multiple Linear Regression (MLR) to identify the correlation between product features and usability. As a result, the Correlation, a matrix is constructed by setting up values for high correlation and moderate correlation as 0.8 and 0.4 respectively. Upon the analysis carried out on disposable razors, it was found that there was no strong correlation between the product feature and usability. For effective product feature usability evaluation, statistical methods such as genetic algorithm, neural network, and fuzzy logic are suggested by the authors for further process.

Wu et al., [1] proposed a web mining approach for product usability evaluation and Feature Fatigue analysis. The customers' reviews are collected from the web using web crawler and preprocessed into review sentences and product feature extraction is performed to form a synonym dictionary. For evaluating the product usability rules are generated by applying FP- growth, frequent itemset algorithm and pruned for reducing the rules. Usability scores and Capability scores are calculated based on the evaluation. In the FF analysis, FF Degree is calculated for each product feature. The result obtained is three features out of seven features were identified with negative FF degree values. This helps the product designers to remove the features with negative values to remove FF of a product.

Wu et al., [23] applied an approach based on review mining for product usability analysis. The analysis is performed by using opinion mining technology for word segmentation and word frequency to convert unstructured review information into structured feature reviews and to identify the semantic orientation of the reviews. In usability evaluation, Kaiser-Meyer-Olkin (KMO) test and Bartlett Test of Sphericity (BTS) is processed for factor analysis to extract feature reviews related to usability. The sentiment scores of product features are calculated to identify the usability and the performance of the product thus helps the manufacturers in developing the least scored product feature.

Long et al., [14] applied web semantic mining method on Chinese customer reviews to analyze product feature usability. The analysis is performed in three steps as a collection of related review words, developing product usability and information system and product feature usability evaluation. In the first step, the word similarity is calculated and related words are identified. In step 2, online reviews are collected using web crawler and analyzed with review words identified in step1 for product usability evaluation. And in step 3, by collecting the Pros and Cons of each feature of the product from the customers, is evaluated. The method was feasible and effective in evaluating total usability score of a product.

Liping et al., [24] proposed Fuzzy Analytic Hierarchy Process (FAHP) to evaluate product usability. In this research work, customers' subjective experience is considered as an important index to identify and evaluate. The usability is derived into three categories as apparent, perceived and performing usability. In FAHP model, fuzzy evaluation matrix is constructed through membership degrees and the values of apparent usability, perceived usability, and performing usability are identified and rated through mean and standard deviation. The model helps to evaluate product usability quantitatively and identifies the product usability problems effectively.

\subsection{Different Existing Methods Applied for Feature Fatigue Analysis}

Chai et al., [25] proposed Norton Bass Model to identify optimal feature combination to increase the Customer Equity (CE). Initially, Norton Bass Model (NBA) is evaluated 
to identify the effects of WOM based on customers' purchase behavior. In the customer transition model, WOM is analyzed based on customers' purchase behavior. And using the customers' purchase analysis, CE is evaluated. Finally, Combinatorial Optimization Problem (COP) is evaluated between capability and usability by applying a genetic algorithm. CE identified is applied as a fitness function for GA evaluation in identifying the optimal feature combination set. This approach increased the CE to about $18 \%$ which helps the manufacturers in improving CE to the product with more features.

Wu et al., [6] in another work proposed an approach based on Kano's model to remove FF. The work initially focuses on identifying the problems in Customer Requirements (CRs) using Continuous Fuzzy Kano's Model (CFKM) to reduce the unwanted features of the product. Then product capability, product usability and FF Index (FFI) are processed in FF analysis. The features with FFI values greater than the threshold value $\mathrm{FFI} \geq(\varphi)=0.10$ is incorporated into the product. Among 11 features only five features are identified with greater values to be added to the product. This helps the product designed to analyze and integrate only the needed features into the product to improve the product quality and as to remove FF.

Wu et al., [20] proposed Bass model for predicting and alleviating feature fatigue of a product. The model is integrated with WOM effects, product usability and capability evaluations identify the impact of product features to be added in product designing. The Customer Equity (CE) is evaluated through the integrated model and analyzed to attain maximum $\mathrm{CE}$ of a product. The model proposed in this work maximizes the $\mathrm{CE}$ for longterm profits as shown in the result of the case study, experimentation the manufacturers gained the maximum CE of $\$ 340.91$. Thus, achieves focus of the work that predicts FF which can support the manufacturers in deciding on adding features to the product and maximizes the CE.

Wu et al., [26] in another work the Bass model is proposed to perform Feature Fatigue (FF) analysis on Customer Equity (CE). The based model analysis is performed based on WOM effects upon usability and capability to identify CE with the product. FF Degree (FFD) is evaluated by identifying the usability score and capability score of each potential feature of the product and ranked. The results achieved in this work helps the manufacturers to consider the values of FF in obtaining $\mathrm{CE}$ and provides support in making decisions on alleviating FF from the product.

$\mathrm{Wu}$ et al., [27] in another work proposed Susceptible-Infected-Recovered (SIR) epidemic model with a genetic algorithm to identify optimal feature combination for increasing the Customer Equity (CE). Initially, the SIR epidemic model is applied to illustrate WOM effects on customers' purchase behavior. And in customer transition model, WOM is analyzed based customers' purchase behavior by different feature combinations. And using the customers' purchase analysis, CE is evaluated. Finally, a genetic algorithm is applied with $\mathrm{CE}$ as a fitness function for evaluation, to identify the optimal feature combination set. The proposed approach increases the CE about 20\%, this helps the product designers in identifying optimal feature combination set to increase CE and remove FF.

Li et al., [28] proposed a Feature Fatigue Multi-Objective Genetic Algorithm (FFMOGA) to solve the problem by adding the features of a product. Initially, the Nondominated Sorting Genetic Algorithm II (NSGA-II) is applied to identify multiple optimal solutions and Bayesian Networks (BNs) fitness function is used to calculate the capability and complexity of each product feature. Finally, features are evaluated by the genetic operators to identify multiple optimal solutions with good performance in convergence.

\section{Proposed Methodology}

In this paper, the DSLC - FOA with Improved FP-Growth Frequent Itemset algorithm is 
used for product usability evaluation and FF degree is computed in feature fatigue analysis.

The reviews about the product and their features posted in online by the customers upon using them are collected using the web crawler tool. To provide the review sentences as input to Association rule mining process, the raw reviews collected from the web are to be preprocessed. The Natural Language Processing (NLP) with Part of Speech (POS) tagging is used for preprocessing. To develop synonym dictionary after preprocessing, Latent Dirichlet Allocation (LDA) and Synonym Lexicon methods are used. The filtering rules are generated to process the extracted features to identify the product features. To evaluate product usability and analyze feature fatigue, DSLC - FOA Algorithm based Association Rule Mining (DFARM) is proposed.

In DFARM, improved FP-Growth frequent Itemset algorithm of Association rule mining approach is improvised to generate a minimum number of optimized rules by analyzing frequent itemsets. The product usability is evaluated by applying those optimized association rules generated on feature related review sentences in the dictionary. Finally, capability evaluation is evaluated in Feature Fatigue (FF) analysis phase. By the determined measures of usability evaluation and capability evaluation, the process of feature fatigue analysis is performed to identify FF degree. This helps in alleviating the feature fatigue effectively.

The framework of the proposed methodology is shown in Figure 1.

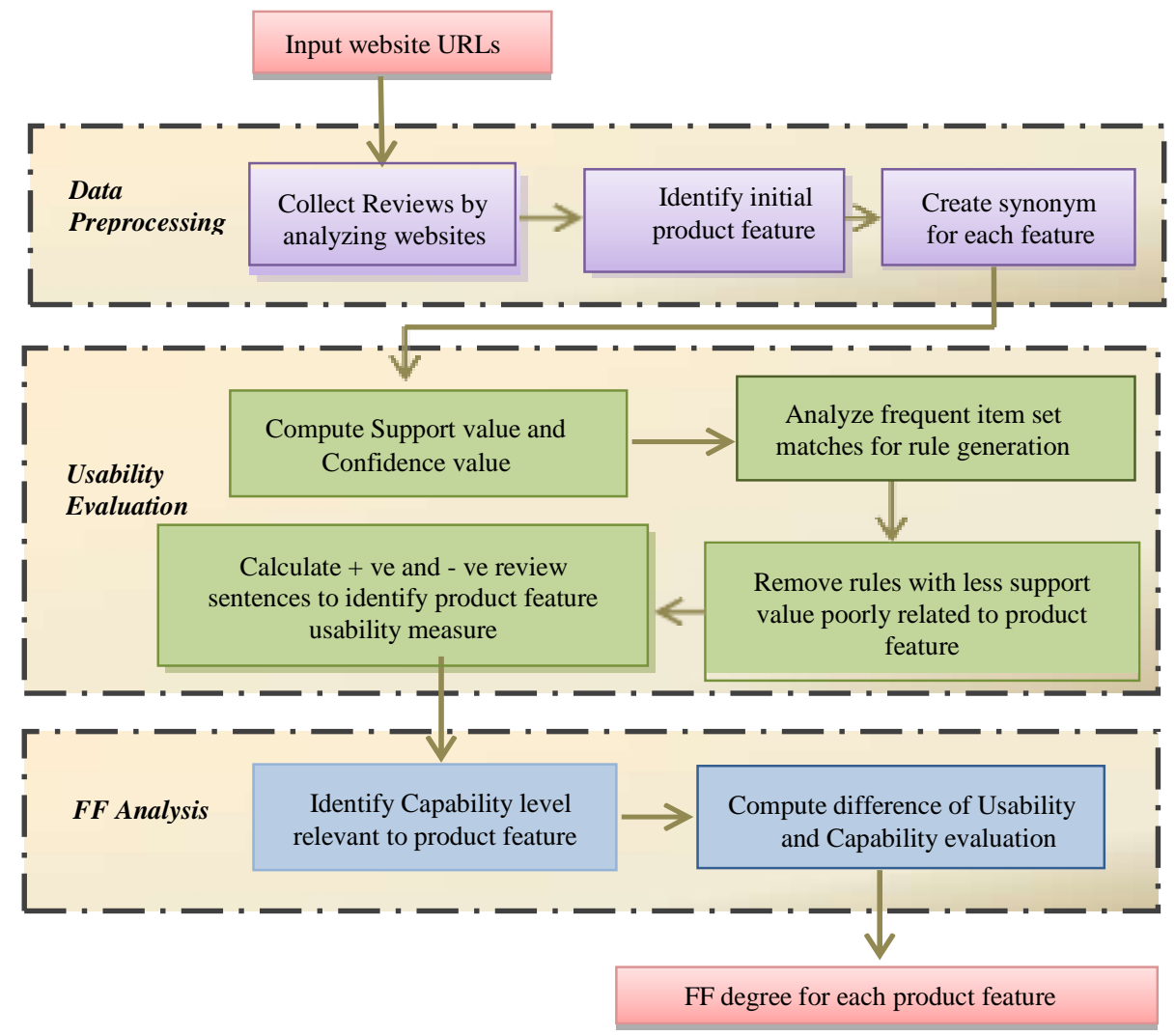

Figure 1. Framework of Proposed DFARM Method

\section{Data Preprocessing}

In this preprocessing phase, the collected raw reviews are converted into review sentences. And using those preprocessed review sentences the synonym dictionary is created. The following sections explain the process of LDA and Lexicon Algorithm in generating the synonym dictionary. In this phase collection of reviews, preprocessing procedure and creation of synonym dictionary are performed. 


\subsection{Collection of Reviews}

The process of collecting reviews is started by collecting the raw reviews of the customers' opinion about the product and its features from various websites using the web crawler tool. The web crawler tool is used to analyze contents in web pages and provides product reviews as output. Those collected raw reviews are stored in review collection database, then preprocessed and converted in to review the sentences. The process of collecting product reviews is demonstrated in the following Figure 2.

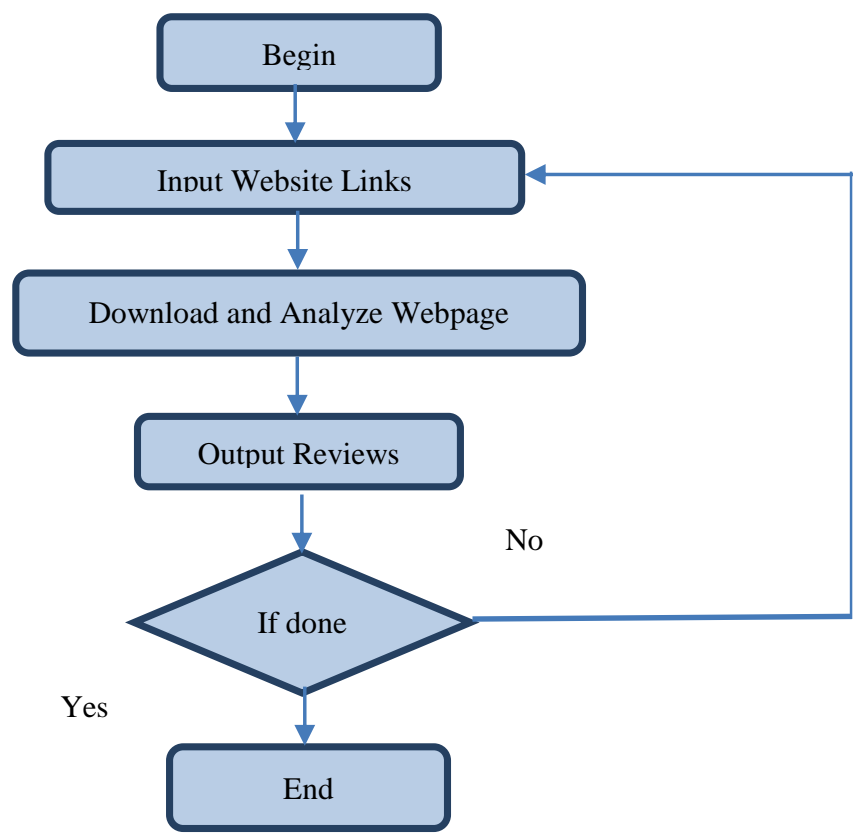

Figure 2. Review Collection Process using a Web Crawler Tool

\subsection{Preprocessing Procedure}

The customers provide the opinions in their own form of words in online about the experience they had on the product and its features. In the reviews posted many words used by the customers may denote to a single and same feature. Identifying the synonyms from such feature is a very difficult process. To analyze and identify such cases Natural Language Processing (NLP) is applied for Part of Speech (POS) tagging on the collected raw reviews. Part-of-speech (POS) tagging is a basic requirement of NLP systems. The process of POS tagging is performed on each review text before considering by LDA model. Part-of-speech tagging (POS tagging) is the method of reviewing a text and parse each word as matching to a specific POS, based on its description such as noun, verb, etc. The POS tagging identifies the initial product feature set of the extracted nouns. POS tagging process is performed on a Sequence of words, $W$ by assigning a sequence of tags, $T$ and computed by identifying the tags which have the maximum frequency in the sequence of words. The process is technically using following equations 1-4.

$$
\begin{aligned}
& P(T \mid W)=P(W \mid T) P(T) / P(W)=\alpha P(W \mid T) P(T) \\
& P(T)=P\left(t_{1}\right) P\left(t_{2} \mid t_{1}\right) \quad P\left(t_{3} \mid t_{1}, t_{2}\right) P\left(t_{3} \mid t_{1}, t_{2}, t_{3}\right) \ldots . P\left(t_{n} \mid t_{1}, t 2 \ldots t_{n-1}\right) \\
& P(W \mid T)=P\left(w_{1} \mid t_{1}\right) P\left(w_{2} \mid t_{2}\right) \ldots . . P\left(w_{n} \mid t_{n}\right) \\
& P(T) P(W \mid T) \approx P\left(t_{1}\right) P\left(t_{2} \mid t_{1}\right) \ldots \ldots . P\left(t_{n} \mid t_{n-1}\right) \quad P\left(w_{1} \mid t_{1}\right) \quad P\left(w_{2} \mid t_{2}\right) \ldots . P\left(w_{n} \mid t_{n}\right)
\end{aligned}
$$

These extracted features are stored in review database and then processed by the LDA 
model to generate the final product feature sets. The synonym dictionary is created by processing the extracted features using LDA and Lexicon algorithm.

\subsection{Creation of Synonym Dictionary}

In this process, for extracting the features from the collected reviews, integrated LDA and lexicon synonym is applied. The process of synonym dictionary is demonstrated in Figure 3.

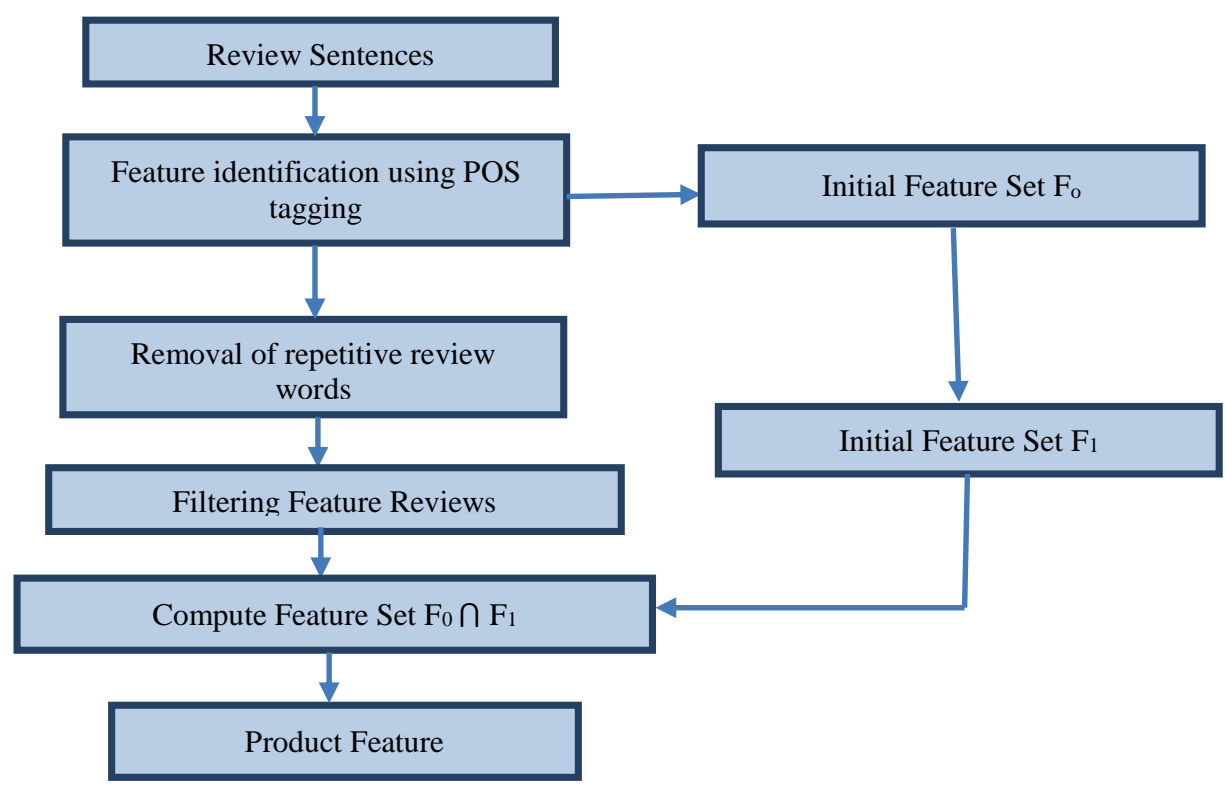

Figure 3. Process of Synonym Dictionary Creation

Table 1 demonstrates the process of LDA and Lexicon Synonym in creating the synonym dictionary.

Table 1. Pseudo Code of LDA with Lexicon Synonym Dictionary

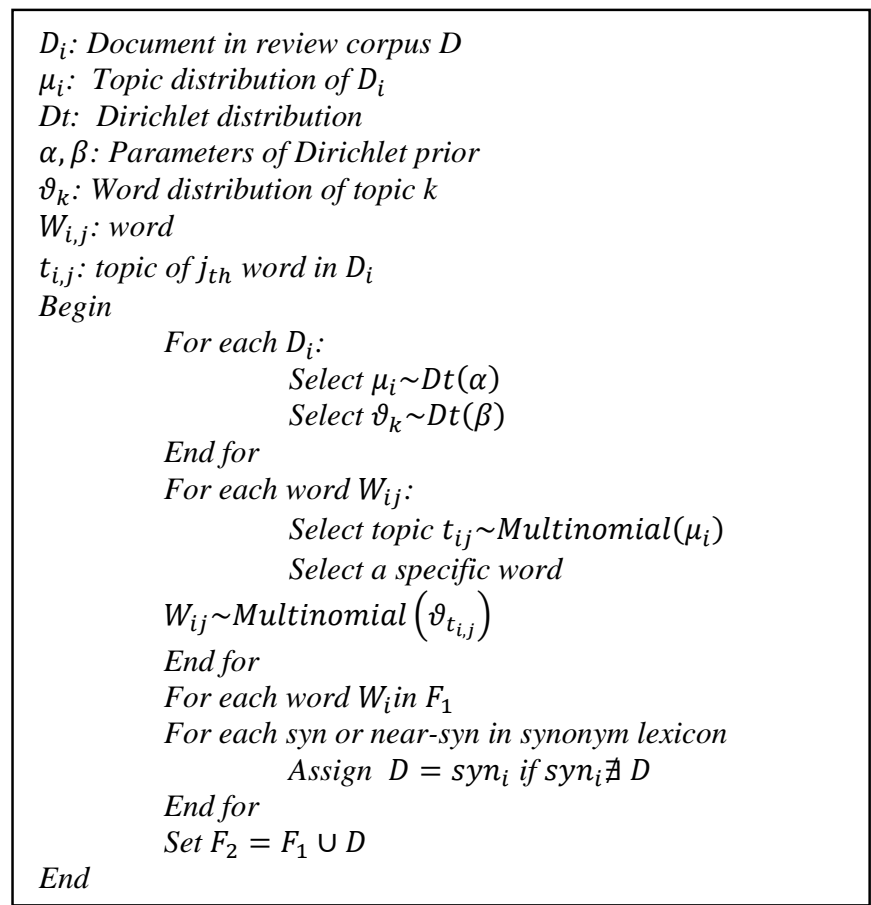


In this phase, the synonym dictionary creation is performed with the help of LDA and Lexicon algorithm. The following section describes the product usability evaluation phase of the proposed contribution.

\section{Product Usability Evaluation}

In this phase, the review sentences are analyzed to generate rules for evaluating the product usability. Initially, the frequent itemset is identified by calculating the support and confidence values of each feature. The obtained frequent itemsets are processed by improved FP-Growth frequent itemset algorithm for generating the association rules. And by applying the DSLC -FOA algorithm rule set reduction is performed to prune the generated rules. Finally, by matching the pruned rules set, product usability is evaluated. Frequent itemset, generating Association Rules, Rule Set Reduction, and usability evaluation processes are performed and explained in the following sections.

\subsection{Frequent Itemset}

For generating the rules by analyzing review sentences the association rule mining is applied. As an association rule mining is the most effective technique in finding the frequent pattern and associations among the information sources, in this work the rule mining is applied to identify the review sentences related to usability features. The problem statement for mining the association rule is, where $l s$ refers to the utmost as shown in equation 5 and 6.

$$
l s=\left\{l s_{1}, l s_{2}, l s_{3}, l s_{4} \ldots . l s_{n}\right\}
$$

And the transaction set as Trans

$$
\text { Trans }=\left\{T_{1}, T_{2}, T_{3}, T_{4} \ldots . T_{n},\right\}
$$

In the process each transaction set, Trans contains the item of an itemset, $l s$. The form of an association rule is $p \emptyset q$, where $p$ and $q$ are the items of $l s, p \cap l s, q \cap l s$ and $p \cap q$. So, when $p$ is contained in the transaction set, and then $q$ will also rely on. An itemset is confirmed as a frequent itemset when its support value is greater than or equal to its threshold value. In this work Memory, RAM, Battery, Processor, etc. are some of the itemsets and clear, better, simple, good, nice, worst, etc. are the items. In the process of identifying association rules, high support items are applied. Based on the transaction percentage the support for the rule is stated and used in transaction $T$. The support of a rule is computed using the following equation 7.

$$
\operatorname{support}(p \rightarrow q)=\frac{p \cup q}{N}
$$

In the above-mentioned equation, $p \cup q$ refers to the total number of transactions of all items of the rule and $N$ represents the total transactions. This computation provides the value of relative support. The confidence value is stated by computing its transaction percentage with $p$ which contains $q$ as shown in below equation 8 .

$$
C(p \Rightarrow q)=\frac{\operatorname{support}(p \rightarrow q)}{\operatorname{support}(p)}
$$

The confidence value computation is a more important measure which views all transactions that contain certain item defined by the rule. The measures computed on the reviews and identified itemsets are processed by improved FP-Growth Frequent Itemset algorithm for generating the rules in the next process.

\subsection{Generating Association Rules}

For mining frequent itemset and learning of association rules, improved FP-Growth 
Frequent Itemset algorithm is applied for effective performance. This process is performed by identifying each individual frequent itemset from the database and analyzes the total number of transactions in the database. Based on the matches between identified frequent itemsets and general trends of the database, association rules are determined. As the FPGrowth Frequent Itemset algorithm processes the analysis repeatedly for identifying the frequent itemset, it has the limitation to perform in many transactions.

In FP-Growth Frequent Itemset the scanning process of the database is repeated for many times to identify the candidate itemsets. This takes more execution time. To overcome this limitation by reducing the execution time in identifying the candidate itemsets, an Improved FP-Growth Frequent Itemset algorithm is applied for processing the itemsets in the review database.

The improved FP-Growth Frequent Itemset algorithm decreases the total number of candidate items in the candidate itemset, $C_{j}$, whereas, in FP-Growth Frequent Itemset algorithm, $C_{j}$ is verified with the support value. If the itemsets are less than the support value, then it is pruned and the itemset $f_{i}$ is produced which relates to itself and lead to $C$. Table 2 shows improved FP-Growth Frequent Itemset algorithm.

\section{Table2. Pseudo Cof Improved FP-Growth Frequent Itemset Algorithm}

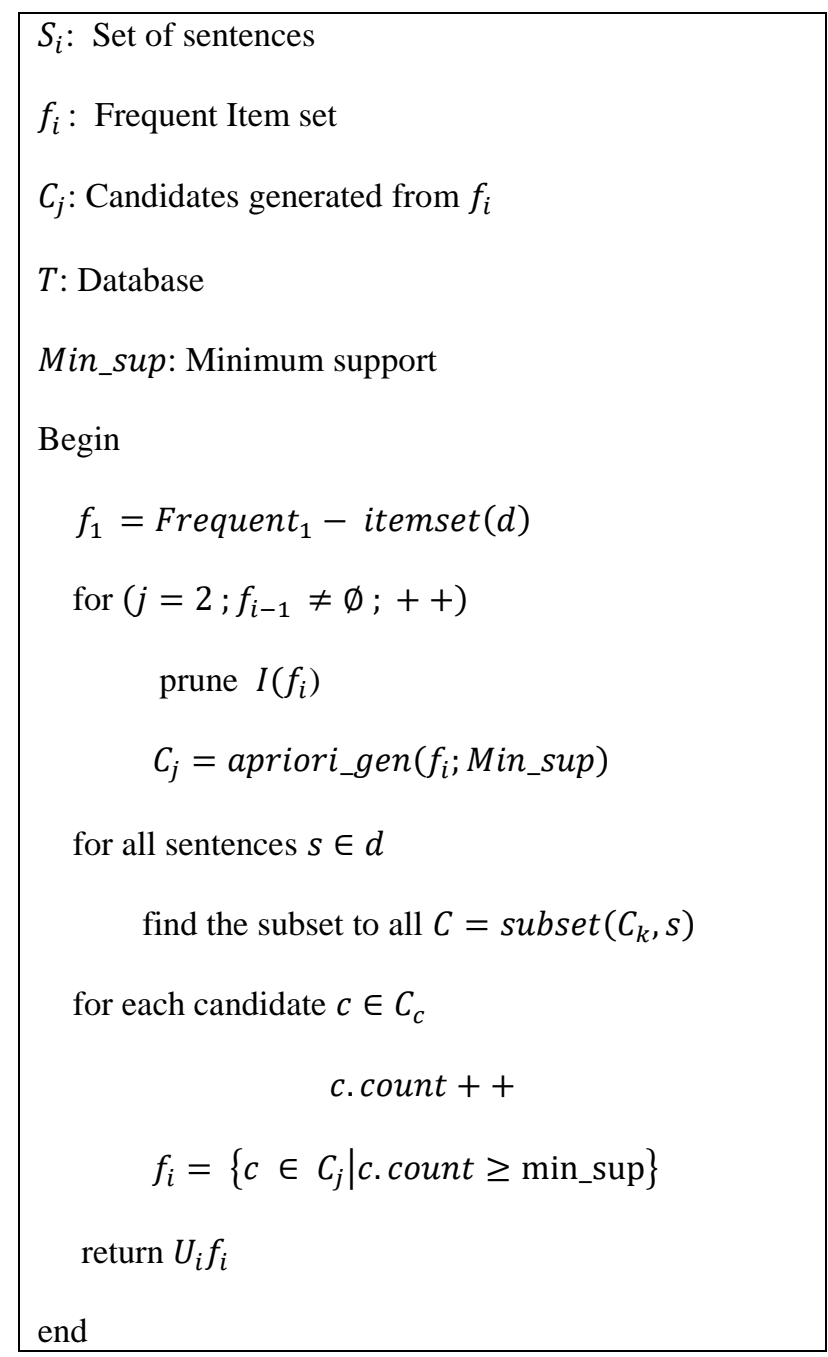

In the improved FP-Growth Frequent Itemset algorithm, before generating the candidate itemsets $C_{j}$ the pruning method counts the times on all items occurred in $f_{i}$ and deletes the itemsets which are less than supportive value. By this improvisation process the number of 
connecting itemsets will be reduced, hence the number of candidate items is also reduced. In the proposed approach, improved FP-Growth Frequent algorithm is applied to obtain the frequent itemset of customer review sentences. A set of sentences $S_{i}=\left(w_{1}, w_{2} \ldots w_{n}\right)$, where $w$ is the words in the $S$. The frequent set of $S_{i}$ is obtained by applying Improved FP-Growth procedure. As a result of applying improved FP-Growth Frequent Itemset algorithm, association rules are generated by evaluating the support value of frequent itemsets identified from product feature review sentences.

\subsection{Rule Set Reduction}

The number of rules generated by improved FP-Growth Frequent Itemset algorithm is reduced by applying DSLC-FOA algorithm. Association rules obtained by improved FPGrowth Frequent Itemset algorithm provided as input for DSLC-FOA technique to identify optimal rules. DSLC-FOA optimization technique analyses the rules and relates to each product feature, based on the support value it removes the rules which has little support values and poorly related to the product feature compared to other rules. This process of removing less concerned rules provides a list of association rules which are more optimized.

In this work, the concept of fruit fly optimization is applied to obtain the product feature set from the synonym dictionary. In the process, the parameters of the synonym dictionary are considered as initial values referred to as Init $X_{\text {axis }}$ and Init $Y_{\text {axis }}$ for computing the association rules to be pruned. Those pruned rules are verified with the support values towards the features and then optimized by removing certain rules with minimum support. The rules which remain are optimized rules considered as input for processing product usability evaluation in the following section. The processing DSLC-FOA algorithm is represented in the following Table 3 .

Table 3. Pseudo Code of DSLC-FOA Algorithm

$$
\begin{aligned}
& \text { Fsl: Fruit fly swarm location } \\
& R v \text { : Random value, Dt: Distance to an origin } \\
& S: \text { Approximate value of Smell concentration } \\
& \text { Smell }_{i}: \text { Smell concentration value } \\
& \text { Fitness_fn: smell concentration judge function } \\
& \text { Begin } \\
& X_{i}=X_{\text {axis }}+R v \\
& Y_{i}=Y_{\text {axis }}+R v \\
& \text { do } \\
& \text { if (food position= unknown) } \\
& \text { find Dt and } S \\
& \begin{array}{l}
D t_{i}=\sqrt{X_{i}^{2}+Y_{i}^{2}} \\
S_{i}=\frac{1}{D t_{i}}
\end{array} \\
& \text { endif } \\
& \text { Substitute } S \text { into fitness_fn } \\
& \text { Smell }_{i}=\text { fitness_fn }_{-}\left(S_{i}\right) \\
& \text { While }\left(\text { Smell }_{i-1}>\text { Smell }_{i}\right) \\
& \text { Assign bestsmell }=\max (\text { Smell }) \\
& X_{\text {axis }}=X(\text { bestindex }) \\
& Y_{\text {axis }}=Y(\text { bestindex }) \\
& \text { End }
\end{aligned}
$$




\subsection{Usability Evaluation}

After the process of rule mining is performed, the optimal rules are included in the new review sentence. This process helps in selecting the rule which matches with the sentences. Based on the confidence value and the support value the classifier identifies the rule that matches with the sentence. The manufacturers can identify and focus on the features with a number of negative usability sentences. But for effective processing, for each product feature, usability level should be identified which can be evaluated using equation 9 as in below.

$$
U n=\alpha\left(n_{N}\right)-n_{p}
$$

Where $n_{N}$ represents the number of negative review sentences, $n_{p}$ represents the numbers positive review sentences and $\alpha$ is the importance level of negative impact related to positive impact. As the measures of product usability measure are displayed in the following Table 4.

Table 4. Product Usability Evaluation Measures

\begin{tabular}{|c|l|}
\hline Measure & \multicolumn{1}{|c|}{ Description } \\
\hline 1 & Strong positive impact \\
\hline 2 & Somewhat strong positive impact \\
\hline 3 & Somewhat weak positive impact \\
\hline 4 & Weak positive impact \\
\hline 5 & Not apparent impact \\
\hline 6 & Weak negative impact \\
\hline 7 & Somewhat weak negative impact \\
\hline 8 & Somewhat strong negative impact \\
\hline 9 & Strong negative impact \\
\hline
\end{tabular}

The process of product usability evaluation is performed as discussed in the above section. And based on customers impact levels product feature usability measure is identified for each feature. These usability measures identified are processed to compute FF degree in FF analysis in the following section.

\section{FF Analysis}

The customers seek for the number of features for the products they buy; this factor may result in dissatisfactory when the feature doesn't fulfill their expectations. This product usability problem is called Feature Fatigue (FF). The focus of this research work is to solve this problem by alleviating FF by analyzing the product usability and capability evaluation measures.

\subsection{Capability Evaluation}

The capability evaluation is also performed as like the measures computed for product usability evaluation. The classified review sentences based on association rules generated are considered on their capability, relevance to the product feature. Based on the impact of the relevant review sentences in the synonym dictionary capability level is measured. The capability measures are obtained by processing the product capability through analyzing the customer reviews and displayed in the following Table 5. These obtained capability measures are to be provided as input with the usability measure obtained in earlier processing to compute FF Degree in the following section. 
Table 5. Product Capability Evaluation Measures

\begin{tabular}{|c|l|}
\hline Measure & \multicolumn{1}{|c|}{ Description } \\
\hline 9 & Extremely attractive \\
\hline 8 & Very attractive \\
\hline 7 & Attractive \\
\hline 6 & Somewhat attractive \\
\hline 5 & Neutral \\
\hline 4 & Somewhat not attractive \\
\hline 3 & Not attractive \\
\hline 2 & Not attractive at all \\
\hline 1 & Extremely not attractive \\
\hline
\end{tabular}

\subsection{FF Degree}

The product capability is evaluated for processing FF analysis in the next step. The obtained measures of product usability and product capability are applied to identify the FF degree. The computation of FF degree is processed using the equation 10.

$$
F F \text { Degree }=U E-C E
$$

In the above formula, the terms $U E$ and $C E$ represents the normalized values of usability and capability measures obtained by computing the equations 11 and 12 .

$$
\begin{aligned}
& U E=\frac{F U E-F U E_{\text {Min }}}{F U E_{\text {Max }}-F U E_{\text {Min }}} \\
& C E=\frac{F C E-F C E_{\text {Min }}}{F C E_{\text {Max }}-F C E_{\text {Min }}}
\end{aligned}
$$

The variable FUE represents the Feature Usability Evaluation. FUE is obtained by evaluating measures obtained in Table 4. In similar, FCE represents the Feature Capability Evaluation which is calculated using the measurements obtained in Table 5. This evaluation process of identifying the value of FF Degree for each feature by applying usability and capability measures provides an analysis of Feature Fatigue. Moreover, it helps manufacturers in alleviating the Feature Fatigue of a product.

\section{Experimental Setup and Results}

In experimentation, the collected raw reviews are converted in to review sentences by preprocessing method and those identified review sentences related to the product features are collected to form a synonym dictionary. By analyzing the review sentences in the dictionary, the association rules are generated, and the numbered rules are reduced by the optimization process. Using those optimized minimum number of association rule product usability and capability measures are evaluated. And the measures obtained are analyzed to find FF degree. The proposed method is implemented using Java. For validation the reviews of the product, Samsung Laptops are collected from an online using web crawler. These raw reviews are the input of this proposed work. To perform the proposed work, 2000 sentences are collected from the web. All those collected sentences are categorized into three sets of sentences as follows.

\section{- $\quad$ A positive attitude toward Usability $P U$ \\ - $\quad$ The negative attitude on Usability $N U$ \\ - $\quad$ Not on Usability $N T$}

Upon processing 260 PUs, 228 NUs and 1312 NTs are classified. In data preprocessing phase, removal of stop words and stemming is performed to extract words that represent 
the product feature. In total there are 50,000 words in the reviews collected. On processing the preprocessing phase 30,212 words are removed Using LDA and Lexicon algorithm synonym dictionary is created. The generated dictionary form is shown in Table 6 below.

Table 6. Generated Synonym Dictionary

\begin{tabular}{|l|l|}
\hline \multicolumn{1}{|c|}{ Feature } & \multicolumn{1}{c|}{ Synonym } \\
\hline Processor & CPU, machine, company, prepares \\
\hline Speed & move quickly, hurry, race, run, sprint, dash \\
\hline Cache & hoard, store, stockpile, stock, supply, collection \\
\hline Memory & recollection, remembrance, reminiscence, evocation \\
\hline RAM & force, thrust, plunge, stab, push, sink, dig, stick, cram \\
\hline Display Features & exhibit, show, put on the show, put on view, layout, set out \\
\hline
\end{tabular}

Using the preprocessed collection of reviews related to the features in the form synonymy dictionary, evaluation of product usability is processed by applying the association rules generated and usability measure is obtained as in Table 7.

Table 7. Product Feature Usability Measure by DFARM Method

\begin{tabular}{|l|c|l|}
\hline \multicolumn{1}{|c|}{ Features } & Measure & \multicolumn{1}{c|}{ Description } \\
\hline Processor & 1 & Strong positive impact \\
\hline Speed & 2 & Somewhat strong positive impact \\
\hline Cache & 2 & Somewhat strong positive impact \\
\hline RAM & 3 & Somewhat weak positive impact \\
\hline Memory & 5 & Not apparent impact \\
\hline Screen size & 7 & Somewhat weak negative impact \\
\hline
\end{tabular}

Similarly, as the product usability evaluation is performed, product capability evaluation is also processed by using the association rule generated by DFARM to analyze the review sentences in a synonym dictionary. Table 8 represents the obtained measure by evaluating the product capability.

Table 8. Result of Capability Evaluation Measure of DFARM Method

\begin{tabular}{|l|c|l|}
\hline \multicolumn{1}{|c|}{ Features } & Measure & \multicolumn{1}{c|}{ Description } \\
\hline Processor & 7 & Attractive \\
\hline Speed & 2 & Not attractive at all \\
\hline Speed & 2 & Not attractive at all \\
\hline Screen size & 4 & Somewhat not attractive \\
\hline Cache & 2 & Not attractive at all \\
\hline RAM & 6 & Somewhat attractive \\
\hline Memory & 7 & Attractive \\
\hline Inbuilt HDD & 3 & Not attractive \\
\hline Optical Drive & 6 & Somewhat attractive \\
\hline Chipset & 9 & Extremely attractive \\
\hline
\end{tabular}

As the measures of product usability and product capability are obtained, feature fatigue analysis is performed by computing the Feature Fatigue Degree (FFD) using those two measures obtained from previous steps. For analyzing Feature Fatigue, the value of FF degree is identified by computing the difference between UE and CE. In this evaluation process, values of FUE and FCE are identified by using the measures obtained in Table 7 and Table 8 . To calculate $U N$, negative review sentences $n_{N}$ and positive review sentences 
$n_{p}$ are used with the $\alpha$ value of 2 are applied and processed. The result of FF analysis computed with results of usability and capability evaluation results is shown in the following Table 9 . The parametric result obtained on evaluating the usability classification and is displayed in Table 10.

Table 9. Result of FF Degree Computation

\begin{tabular}{|l|c|c|c|c|c|}
\hline \multicolumn{1}{|c|}{ Feature } & $\boldsymbol{F U E}$ & $\boldsymbol{F C E}$ & $\boldsymbol{U E}$ & $\boldsymbol{C E}$ & $\boldsymbol{F F}$ Degree \\
\hline Processor & 1 & 8.5 & 0.00 & 0.94 & -0.94 \\
\hline Speed & 2 & 7.25 & 0.00 & 0.78 & -0.78 \\
\hline Cache & 2 & 6.25 & 0.00 & 0.66 & -0.66 \\
\hline RAM & 3 & 7.50 & 0.25 & 0.81 & -0.56 \\
\hline Memory & 5 & 2.50 & 0.50 & 0.19 & 0.31 \\
\hline Screen size & 7 & 6.25 & 0.75 & 0.66 & 0.09 \\
\hline Inbuilt HDD & 7 & 4.75 & 0.75 & 0.47 & 0.28 \\
\hline Optical Drive & 9 & 2.75 & 1.00 & 0.27 & 0.73 \\
\hline Chipset & 9 & 2.50 & 1.00 & 0.19 & 0.81 \\
\hline
\end{tabular}

Table 10. Performance of Product Feature Usability Evaluation for DFARM

\begin{tabular}{|l|c|}
\hline \multicolumn{1}{|c|}{ Performance Metrics } & DFARM Method \\
\hline Precision (\%) & 96 \\
\hline Recall (\%) & 95 \\
\hline F-Measure (\%) & 99 \\
\hline Accuracy (\%) & 99 \\
\hline
\end{tabular}

The results obtained after the experimentation of product usability and FF analysis provides better accuracy as discussed in the above sections. The test analysis report is generated on true positive, true negative, false positive and false negative rate which is used for providing an accurate result in product usability. The values obtained using DFARM method is shown in Figure 4.

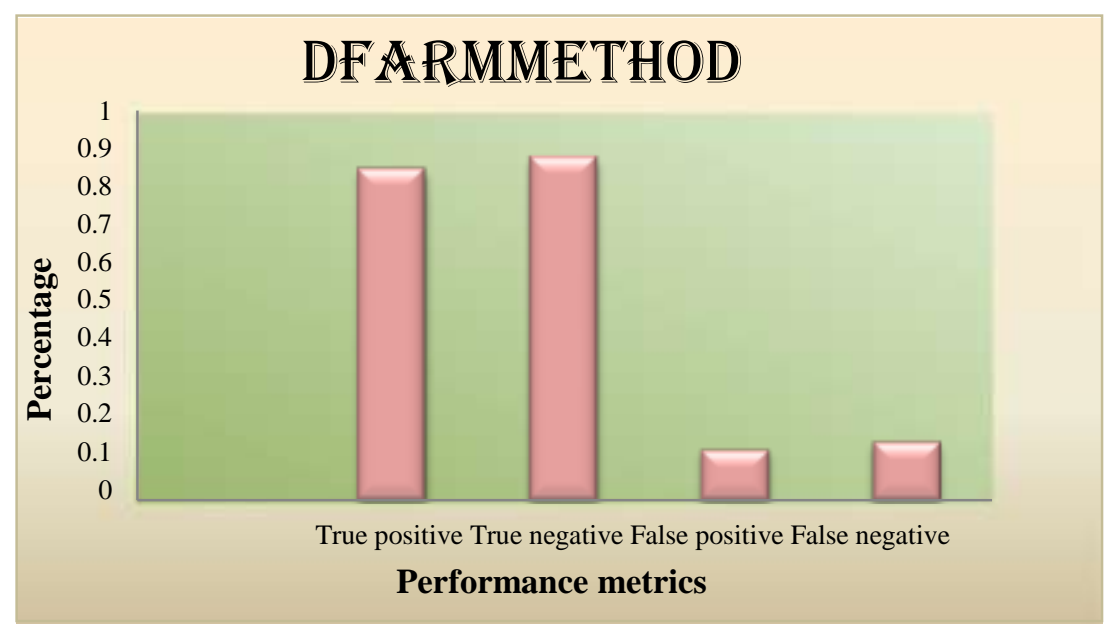

Figure 4. The Result of Test Analysis Report for DFARM

The DFARM method obtains the result in 82 seconds of execution time with $52 \mathrm{Mbps}$ memory utilization. 


\section{Conclusion}

In this paper, the proposed DFARM method with three phases evaluates Product Usability and the capability to analyze Feature Fatigue. The customer reviews are collected through web crawler and preprocessed by applying NLP with POS Tagging. And using LDA with Lexicon Synonym algorithm the synonym dictionary is formed. Then the novel approach, DFARM method is applied to evaluate the review sentences by generating a minimum number of optimized association rules. In the final phase, feature fatigue analysis is performed to identify FF degree. Upon implementing the three phases of experimentation, the results obtained provide better precision value with $96 \%$, Recall value of $95 \%$ and by evaluating the F-Measure the obtained measure is about $99 \%$ which resulted in the usability classifier with $99 \%$ of accuracy. This shows that the proposed contribution helps manufacturers to analyze the usability of product features and helps in making decisions to alleviate Feature Fatigue in product designing.

\section{References}

[1] M. Wu, L. Wang, M. Li and H. Long, "An approach of product usability evaluation based on Web mining in feature fatigue analysis", Computers \& Industrial Engineering, vol. 75, (2014), pp. 230-238.

[2] A. Yee Loong Chong, B. Li, E. WT Ngai, E. Chang and F. Lee, "Predicting online product sales via online reviews, sentiments, and promotion strategies: A big data architecture and neural network approach", International Journal of Operations \& Production Management, vol. 36, no. 4, (2016), pp. 358-383.

[3] B. Li, E. Chang, A. Yee-Loong Chong and H. Bao, "Predicting online e-marketplace sales performances: A big data approach", Computers \& Industrial Engineering, vol. 101, (2016) February 11, pp. 565-571.

[4] X. Ding, B. Liu and P. S. Yu, "A holistic lexicon-based approach to opinion mining", Proceedings of the 2008 international conference on web search and data mining, ACM, (2008), pp. 231-240.

[5] M. Li and L. Wang, "Feature fatigue analysis in product development using Bayesian networks", Expert Systems with Applications, vol. 38, no. 8, (2011), pp. 10631-10637.

[6] M. Wu, L. Wang, H. Long and M. Li, "Feature fatigue analysis in product development", Total Quality Management \& Business Excellence, vol. 26, no. 1-2, (2015), pp. 218-232.

[7] P. Menaka, "A Study on Product Usability Evaluation and Feature Fatigue Analysis Methods for Online Product", Software Engineering and Technology, vol. 9, no. 1, (2017), pp. 7-9.

[8] N. Patel and D. Kumar Singh, "A Survey Based on Product Usability and Feature Fatigue Analysis Methods for Online Product”, Journal of Engineering Computers \& Applied Sciences, vol. 5, no. 8, (2016), pp.53-57.

[9] I. Erkan and C. Evans, "The influence of eWOM in social media on consumers' purchase intentions: An extended approach to information adoption", Computers in Human Behavior, vol. 61, (2016), pp. 47-55.

[10] E. Gide Ergun, and M. X. Wu, "A study for establishing E-commerce Business Satisfaction model to measure e-commerce success in SMEs", International Journal of Electronic Customer Relationship Management, vol. 1, no. 3, (2007), pp. 307-325.

[11] M. Wu, E. Gide and R. Jewell, "The EBS management model: an effective measure of e-commerce satisfaction in SMEs in the service industry from a management perspective", Electronic Commerce Research, vol. 14, no. 1, (2014), pp. 71-86.

[12] J. Midhunchakkaravarthy and S. Selva Brunda, "An Enhanced Web Mining Approach for Product Usability Evaluation in Feature Fatigue Analysis using LDA Model and Association Rule Mining with Fruit Fly Algorithm", Indian Journal of Science and Technology, vol. 9, no. 8, (2016).

[13] X. Li and L. M. Hitt, "Self-selection and information role of online product reviews", Information Systems Research, vol. 19, no. 4, (2008), pp. 456-474.

[14] H. Long, L. Wang and P. Liu, "A method of product feature usability analysis based on web semantic mining”, International Journal of Services Operations and Informatics, vol. 7, no. 2-3, (2012), pp. 136149.

[15] H. Kiso, N. Kashiwase, K. Kasamatsu, M. Yamagishi, H. Jingu and S. Fukuzumi, "Clarification of Kansei elements of attractiveness related to usability for long term mobile phone users", Proceedings of the International Conference of Design, User Experience, and Usability, Springer, Berlin, Heidelberg, (2011), pp. 602-610.

[16] J. Midhunchakkaravarthy and S. SelvaBrunda, "Feature fatigue analysis of product usability using Hybrid ant colony optimization with artificial bee colony approach", Journal of Supercomputing, Springer Science Business Media, (2017).

[17] M. Wu, L. Wang, M. Li and H. Long, "Alleviating feature fatigue in product development based on the bass model”, Journal of Engineering, Design and Technology, vol. 13, no. 3, (2015), pp. 350-368.

[18] Y. Chen, Y. Chai, Y. Liu and Y. Xu, "Analysis of review helpfulness based on consumer perspective", Tsinghua Science and Technology, vol. 20, no. 3, (2015), pp. 293-305. 
[19] M. Zimmermann, E. Ntoutsi and M. Spiliopoulou, "Discovering and monitoring product features and the opinions on them with OPINSTREAM", Neurocomputing, vol. 150, (2015), pp. 318-330.

[20] M. Zubair Asghar, "Lexicon based Approach for Sentiment Classification of User Reviews", Life Science Journal, vol. 11, no. 10, (2014), pp. 468-73.

[21] L. Lizhen, S. Wei, W. Hanshi, L. Chuchu and L. Jingli, "A novel feature-based method for sentiment analysis of Chinese product reviews", China communications, vol. 11, no. 3, (2014), pp. 154-164.

[22] B. Razza and L. Carlos Paschoarelli, "Evaluation of Usability Aspects of Consumer Products in Online Sales", Procedia Manufacturing, vol. 3, (2015), pp. 6237-6244.

[23] M. Wu, L. Wang and L. Yi, "A novel approach based on review mining for product usability analysis", Proceedings of the4th IEEE International Conference of Software Engineering and Service Science (ICSESS), (2013), pp. 942-945.

[24] Y. Li and L. Zhu, "Product usability evaluation method based on fuzzy analytic hierarchy process", Jixie Gongcheng Xuebao(Chinese Journal of Mechanical Engineering), vol. 48, no. 14, (2012), pp. 183-191.

[25] J. Chai, L. Wang, Q. Shi and M. Wu, “Alleviating feature fatigue of multi-generation products”, Industrial Management \& Data Systems, vol. 115, no. 8, (2015), pp. 1435-1456.

[26] M. Wu, L. Wang and M. Li, "An approach based on the Bass model for analyzing the effects of feature fatigue on customer equity”, Computational and Mathematical Organization Theory, vol. 21, no. 1, (2015), pp. 69-89.

[27] M. Wu, L. Wang, M. Li and H. Long, "An approach based on the SIR epidemic model and a genetic algorithm for optimizing product feature combinations in feature fatigue analysis", Journal of Intelligent Manufacturing, vol. 26, no. 1, (2015), pp. 199-209.

[28] M. Li, L. Wang and M. Wu, "A multi-objective genetic algorithm approach for solving feature addition problem in feature fatigue analysis", Journal of Intelligent Manufacturing, vol. 24, no. 6, (2013), pp. 1197 1211. 
International Journal of Advanced Science and Technology

Vol.117 (2018) 